\title{
Evaluation of Secondary Metabolites in Wheat Grain (Triticum Sp.) Grown in Humid South Eastern Plain Zone of Rajasthan (India)
}

\author{
Praveen Kumar Chachaiya ${ }^{1 *}$ and Gargi Mehta ${ }^{2}$
}

${ }^{1}$ Department of Life Science, Career Point University, Kota, Rajasthan, India

${ }^{2}$ Department of Botany, Jiwaji University, Gwalior, Madhya Pradesh, India

*Corresponding author: pkchachaiya@gmail.com (ORCID ID: 0000-0001-7884-8237)

Paper No. 789

Received: 20-04-2019

Revised: 06-07-2019

Accepted: 23-08-2019

\begin{abstract}
Cereal crops such as wheat, rice and barley underpin the staple diet for human consumption, globally. In India, wheat is qualitatively a major source of macromolecule, energy and fiber for human community nutrition since long time hence, preferably used as a staple food grain for society and also used as major source of fodder for animal feeding. The health benefits of whole grains are linked to the existence of secondary bioactive metabolites including phenolic acids, flavonoids and phytosterols. Flavonoids have the properties like anti-inflammatory, antioxidant, antiallergic, antithrombotic, antiviral and anticarcinogenic activities. Quercetin is one with an average of daily consumption of $25 \mathrm{mg}$ to $40 \mathrm{mg}$. Kaempferol used as an antioxidant. Quantification data revealed that the total flavonoid content (free + bound) was observed highest and lowest in durum genotypes of HD 4728 (1.75 mg/gdw) and Raj 6560 (0.92 mg/gdw), respectively and in aestivum genotypes Raj 4037 (1.38 mg/gdw) and HI 1544 (0.72 mg/ $\mathrm{gdw})$ gave similar trend. Regular daily intakes of whole grain products are associated with reduced risk of several diseases. The objective of this study was identification and characterization of secondary metabolites i.e. flavonoids in Triticum sp.

Highlights

( Quantification study of secondary metabolites (flavonoid compounds) in 2 wheat species, standard samples were analyzed by GC-MS, Flavonoid's have the strongest antioxidant properties.
\end{abstract}

Keywords: Triticum, Secondary metabolite, Flavonoid, TLC, GC-MS

Wheat is a herbaceous plant and monocotyledonous member of Gramineae family. Probably, it's the First plant that is domesticated and cultivated by human. The Indian wheat is predominantly mature and known for creating tasty and quality Chapattis. The abnormal amplify in area, production and productivity of wheat crop, established newer higher records with each passing year. Wheat is a big concerning 29 million hectare acreage of everywhere in the India (J. Kumar et al., 2016), used mainly for human consumption to support approximate 35\% population of the world (Mohammadi-joo et al. 2015) and also provide 20 per cent of the total food calories (Anonymous, 2014).
According to United Nations DESA (Department of Economic and Social Affairs) report, World population is expected to increase from around 7 billion now to around 8.5 billion in the year 2030 . In South Asia and continent, 2 out of 5 kids may still starving despite, distinct improvement in per caput food accessibility. Thus, food and nutritional security will continue to remain major challenge for developing countries in the world.

Based on rainfall, soil characterization, temperature etc. wheat cultivation is divided in to six mega zones of India (Map-I). The biggest state Rajasthan consisting 10.4 per cent of total geographical region 
and 5.67 per cent of total population of the Republic of India (Census, 2011). In Agro climatic zones, India has been portrayed into 126 agro-climatic zones by the Indian Council of Agricultural Research (ICAR), out of that Rajasthan has been bifurcate into ten agro-climatic zones. In agricultural regions of Rajasthan factor's like rainfall, altitude, latitude, temperature, topography, natural vegetation, soils, crops, irrigation availability and livestock are taken into consideration. Humid South Eastern Plain Zone covers the area of Kota, Bundi, Baran, Jhalawar and some parts of Sawai Madhopur districts of Rajasthan state in India.

Generally plant produces two types of metabolites i.e. primary metabolites and secondary metabolites. Primary metabolites directly concerned in growth and metabolism of plant. Secondary metabolites are considered as end product of primary metabolites that have no major role in the maintenance of plant, but they are extremely important compounds from the biological point of view. They are the speciesspecific and are formed only in certain tissue, cell or organ (Pichersky and Gang 2000). Plant secondary metabolites i.e. phytochemicals etc. are an important sources for pharmaceutical, that contribute medical properties of plant. They provide the flavors, specific odors and colors in plants. They are found in cereals either as free or bound molecules (Dykes and Rooney 2007).

Secondary metabolites includes alkaloids, glycosides, gums, flavonoids, coumarins, polysaccharides, tannins, terpenes, phenols and terpenoids and formed in plants ordinary metabolic processes (Harborne 1973; Okwu 2004). Flavonoids are one of the important group of secondary metabolites, its, water soluble phenolic glycosides imparting colour to flowers and fruits of higher plants. Flavonoids also contribute in the physiological functions such as seed maturation and dormancy (Brenda 1998). These are bioactive compounds with more than 9000 structural variants known (Williams and Grayer, 2004). According to (Peer and Murphy 2007) one of their most important roles is to influence the transport of plant hormone auxin. These types of compounds also appear for play vital roles to defense plants against pathogens and predators (Winkel-Shirley 2002). It also have the properties like anti-inflammatory, antioxidant, antiallergic, antithrombotic, antiviral and anticarcinogenic activities (Middleton and Kandaswami 1993, Williams and Grayer 2004; Garcia - Mediavilla et al. 2007; Pandey et al. 2007; Kim et al. 2008; Singh et al. 2008; Sharma and Sarin 2012c; 2012d).

Juice of wheatgrass may be used for increasing production of haemoglobin in blood, improving wound healing, prevention of tooth decay and prevention of bacterial infections. It should conjointly used as removing of significant metals, medicine and cancer-causing agents from the body and also for removing toxins from the liver and blood (MacIntosh 2008). Many more research has been done on secondary metabolites available in wheat grass juice but there are lacks of research in wheat grain. So, this research interest is made on that.

\section{MATERIALS AND METHODS}

The experiment was conducted at Dhakarkheri village, Kaithoon road, Kota (Rajasthan) situated in between $25^{\circ} 11^{\prime} \mathrm{N}$ latitude and $75^{\circ} 54^{\prime} \mathrm{E}$ longitudes at $273 \mathrm{~m}$ altitude from mean sea level, during two consecutive rabi season 2015-16 and 2016-17. In this study seeds of 2 wheat species 10 genotypes (Recommended for the region) in which 5 genotypes each of species Triticum aestivum L. (Raj 4037, Raj 4238, GW 322, GW 366, HI 1544) and Triticum durum desf. (Raj 6560, MPO 1215, HI 8498, HI 8737, HD 4728) was sown in Randomized Block Design with three replications.

\section{Isolation, Identification and Quantification of Secondary Metabolite (Flavonoids)}

\section{Extraction}

Seeds of studied wheat genotypes were air dried and grind fine powdered separately. All the genotypes seed samples was extracted separately with $80 \%$ methanol on water bath (Subramanian and Nagarajan 1969) for 24 hrs. In the beginning methanol soluble fractions were filtered then concentrated in vacuo and aqueous fractions were later fractioned by sequential extraction with petroleum ether (Fr I), diethyl ether (Fr II) and ethyl acetate (Fr III), separately. Every process was repeated three times for complete extraction; fraction I was discarded in each process because it contain fatty substance, whereas fraction II 
and fraction III were concentrated and used for evaluation of flavonoids.

Fraction III was hydrolyzed by refluxing with $7 \%$ Sulphuric acid $\left(\mathrm{H}_{2} \mathrm{SO}_{4}\right)\left(10 \mathrm{mLg}^{-1}\right.$ plant material for 2 hrs.) filtered and filtrate was extracted three times with ethyl acetate. All ethyl acetate layers were pooled separately, neutralized by distilled water with repeated washings and concentrated in vacuo. Both, fraction II and III were taken up in a little volume of ethanol (2-5 $\mathrm{mL}$ ) before chromatographic investigations.

\section{Qualitative procedure}

\section{Thin Layer Chromatography (TLC)}

$20 \times 20 \mathrm{~cm}$ thin glass plates were coated with 250 $\mathrm{m} \mu$ thick Silica gel G. Newly prepared gel plates then air dried at room temperature; thereafter these TLC plates were kept at $100^{\circ} \mathrm{C}$ for $1 / 2$ hrs to activate and then cooled at room temperature. These silica gel plates may be used for the determination.

Every extracted sample was co-chromatographed with an authentic flavonoid as marker (quercetin and kaempherol). Ready TLC plates were developed in an air tight chromatographic chamber and then saturated with solvent mixture (Benzene: Acetic Acid: Water :: 125:72:3; Wong and Francis, 1968). Developed gel plates were air dried and visualized under UV light by exposure to ammonia fumes. The mouth of a $100 \mathrm{~mL}$ containing concentrated $\mathrm{NH}_{4} \mathrm{OH}$ was held in contact with each spot for about 5-10 seconds and fluorescent spots corresponding to that of standard markers were marked. Thereafter, gel plates sprayed with $5 \% \mathrm{FeCl}_{3}$ and $0.1 \%$ alcoholic $\mathrm{AlCl}_{3}$ kept in an $\mathrm{I}_{2}$ (Iodine) chamber, separately. Colored spots developed to be noted and the Rf value of each spot was then calculated and computed. Many other such type of solvent systems like n-butanol: acetic acid: water :: 4:1:5; tertiary butanol: acetic acid: water :: 3:1:1 were also used for this purpose, but the solvent system benzene: acetic acid: water :: 125:72:3 gave the appropriate and suitable result.

\section{GC-MS Profiling}

The extract and standard samples were analyzed by GC-MS (Gas Chromatography and Mass Spectroscopy) of Hewlett-Packard 6890/5973 operating at $1000 \mathrm{ev}$ ionization energy, equipped with using Agilent 7890A/ 5975C GC HP-5. Containing capillary column (phenyl methyl siloxane, $25 \mathrm{~m} \times 0.25 \mathrm{~mm}$ i.d) with Helium $(\mathrm{He})$ was used as carrier gas with flow of $(0.9 \mathrm{~mL} / \mathrm{min})$ and split ratio of $1: 5$. Oven temperature was $100^{\circ} \mathrm{C}(3$ $\mathrm{min}$ ) to $280^{\circ} \mathrm{C}$ at 1 to $40^{\circ} \mathrm{C} / \mathrm{min}$; detector temperature $250^{\circ} \mathrm{C}$ to $280^{\circ} \mathrm{C}$. Retention indices were determined by using retention times (RT) of samples that were injected under the same chromatographic conditions. All components of the standard and plant samples were justified by comparison of their mass spectra and retention time with those given in literature and by comparison with the mass spectra of Wiley library or by the published mass spectra.

\section{RESULTS AND DISCUSSION}

Spot of flavonoids were observed in sample of wheat species viz; Triticum aestivum L. and Triticum durum desf. by thin layer chromatography (TLC) plates prepared and sprayed with $5 \% \mathrm{FeCl}_{3}$. The $\mathrm{R}_{\mathrm{f}}$ value of the spots matched with their authentic standards and then identified as quercetin and Kaempferol. Solvent system Benzene: Acetic Acid: Water (125:72:3) gave best results with $R_{f}$ values viz; quercetin 0.78 and Kaempferol 0.83 (Table 2). Availability of flavonoid compounds was confirmed with thin layer chromatography (TLC) and Gas chromatography and mass spectrometry (GC-MS). The identification and isolation of different flavonoids (Quercetin and Kaempferol) was done by some common measurements i.e. $\mathrm{mp}, \mathrm{mmp}$, performed in capillaries (Toshniwal freezing Point Apparatus), IR (Infra-red spectrophotometer; Perkin, Elmer 337, Grating Infrared spectrophotometer) UV (Ultraviolet and visual spectrophotometer; Carl Zeiss, Jena, DDR, VSUZP spectrophotometer) analysis along with their several authentic samples. TLC plates proved that two fluorescent spots coinciding are as authentic standard of quercetin and Kaempferol. Further identification was done by $\mathrm{R}_{\mathrm{f}}$ values (quercetin 0.78 and Kaempferol 0.83), melting point (Quercetin $309-311^{\circ} \mathrm{C}$, Kaempferol $271-273^{\circ} \mathrm{C}$ ) and the colour reaction tests, when sprayed with $5 \%$ ethanolic $\mathrm{FeCl}_{3}$ (Quercetin: green-yellow, Kaempferol: yellowbrown). The characteristic IR peaks of isolated flavonoids were found to be super impossible with their respectable standard quercetin and kaempferol. 
Table 1: Flavonoid contents (free + bound) in 10 genotypes of Triticum species

\begin{tabular}{ccccccccccc}
\hline $\begin{array}{c}\text { Sl. } \\
\text { No. }\end{array}$ & $\begin{array}{c}\text { Wheat } \\
\text { Genotypes }\end{array}$ & \multicolumn{2}{c}{ Free flavonoids (mg/gdw) } & \multicolumn{3}{c}{$\begin{array}{c}\text { Bound flavonoids } \mathbf{( m g} / \\
\text { gdw) }\end{array}$} & $\begin{array}{c}\text { Total } \\
\text { Kaempferol } \\
\text { (mg/gdw) }\end{array}$ & $\begin{array}{c}\text { Total } \\
\text { Quercetin } \\
\text { (mg/gdw) }\end{array}$ & $\begin{array}{c}\text { Total flavonoids } \\
\text { (free+bound ) } \\
\text { (mg/gdw) }\end{array}$ \\
\hline & & $\mathbf{Q}$ & $\mathbf{K}$ & $\mathbf{T}$ & $\mathbf{Q}$ & $\mathbf{K}$ & $\mathbf{T}$ & \\
\hline 1 & Raj 4037 & 0.35 & 0.76 & 1.11 & 0.03 & 0.24 & 0.27 & 1.0 & 0.38 & 1.38 \\
2 & Raj 4238 & 0.29 & 0.65 & 0.94 & 0.02 & 0.12 & 0.14 & 0.77 & 0.31 & 1.08 \\
3 & GW 322 & 0.13 & 0.44 & 0.57 & 0.09 & 0.36 & 0.45 & 0.8 & 0.22 & 1.02 \\
4 & GW366 & 0.18 & 0.44 & 0.62 & 0.14 & 0.36 & 0.50 & 0.8 & 0.32 & 1.12 \\
5 & HI1544 & 0.09 & 0.30 & 0.39 & 0.05 & 0.28 & 0.33 & 0.58 & 0.14 & 0.72 \\
6 & Raj 6560 & 0.18 & 0.33 & 0.51 & 0.12 & 0.29 & 0.41 & 0.62 & 0.3 & 0.92 \\
7 & MPO1215 & 0.22 & 0.47 & 0.69 & 0.06 & 0.32 & 0.38 & 0.79 & 0.28 & 1.07 \\
8 & HI 8498 & 0.37 & 0.78 & 1.15 & 0.10 & 0.35 & 0.45 & 1.13 & 0.47 & 1.60 \\
9 & HI 8737 & 0.29 & 0.64 & 0.93 & 0.08 & 0.55 & 0.63 & 1.19 & 0.37 & 1.56 \\
10 & HD 4728 & 0.43 & 0.85 & 1.28 & 0.11 & 0.36 & 0.47 & 1.21 & 0.54 & 1.75 \\
\hline
\end{tabular}

Abbreviations: Q: Quercetin, K: Kaempferol, T: Total.

Table 2: Chromatographic data and colour reaction of the flavonoids isolated from seed of Triticum species

\begin{tabular}{cccccccc}
\hline \multirow{2}{*}{ Flavonoids } & \multicolumn{2}{c}{$\mathbf{R f}(\times \mathbf{1 0 0})$ in BeAW+ } & \multicolumn{2}{c}{ Colour reaction } & \multirow{2}{*}{ MP'C $^{\circ}$} & \multirow{2}{*}{ UV maximum $^{\prime}$} \\
\cline { 2 - 5 } & Standard & Seed & Day- light & $\mathbf{I}_{2}$ vapours & & \\
\hline Quercetin & 78 & 0.77 & GN-YW & YW-BN & $309-311$ & 258,373 \\
Kaempferol & 83 & 0.81 & GN-YW & YW-BN & $271-273$ & 268,368 \\
\hline
\end{tabular}

Abbreviations: ${ }^{+B e} A W=$ Benzene: Acetic acid: Water (125: 72:3); BN = Brown; $G N=$ green; $Y W=$ yellow.

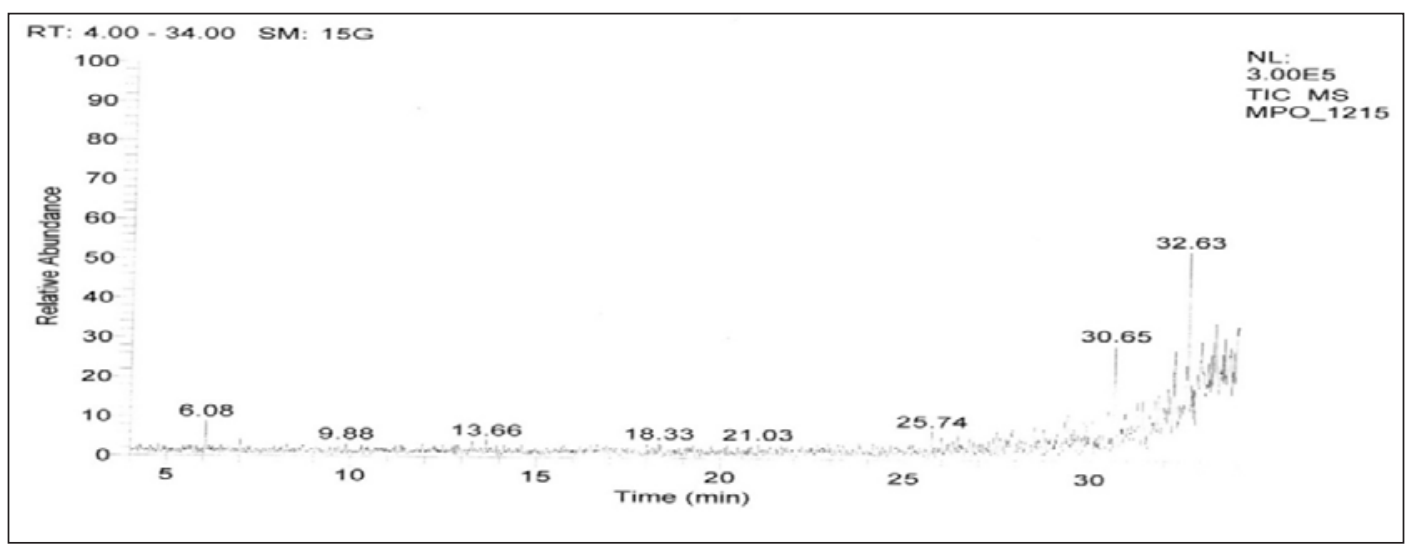

Fig. 1: GC-MS analysis of Triticum species genotype MPO 1215

In the most excessive dietary flavonoids quercetin is one with an average of daily consumption of $25 \mathrm{mg}$ to $40 \mathrm{mg}$ (Formica and Regelson 1995). Kaempferol is a secondary substance that is type of flavonoid commonly used in several plants, plant-derived foods and ancient medicines (Calderon-Montano et al. 2011). It is used as an antioxidant. Ancient research studies recommend that consuming kaempferol may decrease the risk of many types of cancers but currently, it is under the contemplation for possible cancer treatment.
It was observed that in GC-MS profiling of flavonoids, total 50 compounds each were found for 4 best among 10 studied genotypes of Triticum species. The spectra revealed that major compound present in seed of Triticum sp. were Butyl Isobutyl Isobutal (9.81\%), followed by Phenyltetrafluorophosphorane (7.52\%), Oxalic acid, monoamide, n-propyl, tridecyl ester (6.73\%), Dichloroacetic acid, 2,2-dimethylpropyl ester (5.02\%) and Propane,1-(2,2-dichloro-1methylcyclopropyl)-2,2-dimethyl (5.02\%) in MPO 1215 genotype (Table 3, Fig. 1). 
Table 3: Phytocompounds identified by GC-MS in seed extract of durum wheat genotype (MPO 1215)

\begin{tabular}{|c|c|c|c|c|c|}
\hline $\begin{array}{c}\text { S1. } \\
\text { No. }\end{array}$ & RT & Compound Name & $\begin{array}{l}\text { Molecular } \\
\text { Formula }\end{array}$ & MW & $\begin{array}{c}\text { Area } \\
(\%)\end{array}$ \\
\hline 1 & 6.08 & $\begin{array}{l}\text { 4H-1-Benzopyran-4-one, 3,8-dihydroxy-5,6,7-trimethoxy-2-(4- } \\
\text { methoxyphenyl)- }\end{array}$ & $\mathrm{C}_{19} \mathrm{H}_{18} \mathrm{O}_{8}$ & 374 & 0.83 \\
\hline 2 & 25.74 & 6-Ethyl-4,5,7,8-tetrathia-2-nonene & $\mathrm{C}_{7} \mathrm{H}_{14} \mathrm{~S}_{4}$ & 226 & 0.61 \\
\hline 3 & 26.44 & Cyclohexane, 1,4-dichloro-, cis- & $\mathrm{C}_{6} \mathrm{H}_{14} \mathrm{C}_{12}$ & 152 & 0.45 \\
\hline 4 & 27.49 & 2-(tert-Butylsulfonyl)-N'-hydroxyethanimidamide & $\mathrm{C}_{6} \mathrm{H}_{14} \mathrm{~N}_{2} \mathrm{O}_{3} \mathrm{~S}$ & 194 & 0.69 \\
\hline 5 & 27.90 & $\begin{array}{l}\text { Isoindole-1,3-dione, 1,3-dihydro-5-(3-aminophenoxy)-2-(4- } \\
\text { aminophenyl)- }\end{array}$ & $\mathrm{C}_{20} \mathrm{H}_{15} \mathrm{~N}_{3} \mathrm{O}_{3}$ & 345 & 1.83 \\
\hline 6 & 28.49 & Pentane-1,3-diol dipropionate, 2-methyl- & $\mathrm{C}_{12} \mathrm{H}_{22} \mathrm{O}_{4}$ & 230 & 0.66 \\
\hline 7 & 28.74 & 1-Butanol, 2-methyl-, propanoate & $\mathrm{C}_{8} \mathrm{H}_{16} \mathrm{O}_{2}$ & 144 & 0.79 \\
\hline 8 & 29.30 & Acetic acid, trifluoro-, 2,2-dimethylpropyl ester & $\mathrm{C}_{7} \mathrm{H}_{11} \mathrm{~F}_{3} \mathrm{O}_{2}$ & 184 & 1.22 \\
\hline 9 & 29.39 & 1,3,2-Dioxathiolane, 4-methyl-, 2-oxide & $\mathrm{C}_{3} \mathrm{H}_{6} \mathrm{O}_{3} \mathrm{~S}^{2}$ & 122 & 1.41 \\
\hline 10 & 29.48 & Peroxide, dibutyl & $\mathrm{C}_{8}^{3} \mathrm{H}_{18}^{6} \mathrm{O}_{2}$ & 146 & 0.36 \\
\hline 11 & 29.55 & Cyclopropaneethanol, 2-iodo- & $\mathrm{C}_{5}^{8} \mathrm{H}_{9} \mathrm{IO}$ & 212 & 0.49 \\
\hline 12 & 29.66 & Pentanoic acid, 1,1dimethylpropyl ester & $\mathrm{C}_{10} \mathrm{H}_{20} \mathrm{O}_{2}$ & 172 & 0.67 \\
\hline 13 & 29.84 & 5-Amino-7-methylaminofurazano[3,4-d]pyrimidine & $\mathrm{C}_{5} \mathrm{H}_{6} \mathrm{~N}_{6} \mathrm{O}$ & 166 & 0.33 \\
\hline 14 & 29.89 & Tert-Butyl methyl carbonate & $\mathrm{C}_{6} \mathrm{H}_{12} \mathrm{O}_{3}$ & 132 & 0.82 \\
\hline 15 & 30.04 & Propanamide, 2,3,3,3-tetrafluoro-2-trifluoromethoxy- & $\mathrm{C}_{4} \mathrm{H}_{2} \mathrm{~F}_{7} \mathrm{NO}_{2}$ & 229 & 1.92 \\
\hline 16 & 30.24 & 1-(2-Phenylsulfanyl-ethyl)-pyrrolidine & $\mathrm{C}_{12} \mathrm{H}_{17} \mathrm{NS}$ & 207 & 0.35 \\
\hline 17 & 30.48 & $\begin{array}{l}\text { 4-((5-Ethenyl-1-azabicyclo( } 2,2,2) \text { octan-2-yl)oxymethyl)-6-methoxy- } \\
\text { quinoline }\end{array}$ & $\mathrm{C}_{20} \mathrm{H}_{24} \mathrm{~N}_{2} \mathrm{O}_{2}$ & 324 & 2.31 \\
\hline 18 & 30.65 & Oxalic acid, butyl cyclobutyl ester & $\mathrm{C}_{10} \mathrm{H}_{16} \mathrm{O}_{4}$ & 200 & 4.84 \\
\hline 19 & 30.91 & Pentane, 1bromo3,4dimethyl & $\mathrm{C}_{7} \mathrm{H}_{13} \mathrm{Br}$ & 178 & 1.10 \\
\hline 20 & 30.95 & Dichloroacetic acid, 2,2dimethylpropyl ester & $\mathrm{C}_{7} \mathrm{H}_{12} \mathrm{C}_{12} \mathrm{O}_{2}$ & 198 & 1.02 \\
\hline 21 & 31.26 & Carbonic acid, neopentyl cyclohexylmethyl ester & $\mathrm{C}_{13} \mathrm{H}_{2} \mathrm{O}_{3}$ & 228 & 1.28 \\
\hline 22 & 31.40 & Trifluoroacetyl-di-tbutylphosphine & $\mathrm{C}_{10} \mathrm{H}_{18} \mathrm{~F}_{3} \mathrm{OP}$ & 242 & 2.53 \\
\hline 23 & 31.59 & Sulfurous acid, isobutyl pentyl ester & $\mathrm{C}_{9} \mathrm{H}_{20} \mathrm{O}_{3} \mathrm{~S}$ & 208 & 1.44 \\
\hline 24 & 31.68 & 5-Hepten-3-one, 5-ethyl-2-methyl- & $\mathrm{C}_{10} \mathrm{H}_{18} \mathrm{O}$ & 154 & 0.86 \\
\hline 25 & 31.74 & 2,2-Dimethyl-propyl 2,2-dimethyl-propanesulfinyl sulfone & $\mathrm{C}_{10} \mathrm{H}_{22} \mathrm{O}_{3} \mathrm{~S}_{2}$ & 254 & 2.19 \\
\hline 26 & 31.83 & 1nButoxy2,3dimethyldiaziridine & $\mathrm{C}_{8} \mathrm{H}_{17} \mathrm{NO}$ & 143 & 1.86 \\
\hline 27 & 31.98 & 2-Hexene,1-(pentyloxy)-,(E)- & $\mathrm{C}_{11} \mathrm{H}_{22} \mathrm{O}$ & 170 & 1.25 \\
\hline 28 & 32.04 & 2-Hexenoic acid, 4-methylphenyl ester & $\mathrm{C}_{13} \mathrm{H}_{16} \mathrm{O}_{2}$ & 204 & 0.96 \\
\hline 29 & 32.09 & 2,4Dimethylpentan3yl isobutyl carbonate & $\mathrm{C}_{11}^{13} \mathrm{H}_{22} \mathrm{O}_{3}$ & 202 & 1.83 \\
\hline 30 & 32.15 & Ethane, 1,2-dicyclopropyl- & $\mathrm{C}_{8} \mathrm{H}_{14}$ & 110 & 1.99 \\
\hline 31 & 32.22 & 3-Methylbutyl N-(heptafluorobutyryl)isoleucinate & $\mathrm{C}_{15} \mathrm{H}_{22} \mathrm{~F}_{7} \mathrm{NO}_{3}$ & 397 & 3.09 \\
\hline 32 & 32.27 & Sulfurous acid, butyl hexyl ester & $\mathrm{C}_{10} \mathrm{H}_{22} \mathrm{O}_{3} \mathrm{~S}$ & 222 & 4.66 \\
\hline 33 & 32.39 & Peroxide, dibutyl & $\mathrm{C}_{8} \mathrm{H}_{18} \mathrm{O}_{2}$ & 146 & 0.50 \\
\hline 34 & 32.63 & Butyl Isobutyl Isobutal & $\mathrm{C}_{12} \mathrm{H}_{26} \mathrm{O}_{2}$ & 202 & 9.81 \\
\hline 35 & 32.71 & Cis-1-methyl-3-n-nonylcyclohexane & $\mathrm{C}_{16} \mathrm{H}_{32}$ & 224 & 1.25 \\
\hline 36 & 32.75 & Acetic acid, trifluoro-, 2,2-dimethylpropyl ester & $\mathrm{C}_{7} \mathrm{H}_{11} \mathrm{~F}_{3} \mathrm{O}_{2}$ & 184 & 1.00 \\
\hline 37 & 32.80 & 2,2-Dimethyl-propyl 2,2-dimethyl-propanesulfinyl sulfone & $\mathrm{C}_{10} \mathrm{H}_{22} \mathrm{O}_{3} \mathrm{~S}_{2}^{2}$ & 254 & 1.31 \\
\hline 38 & 32.88 & Dichloroacetic acid, 2,2-dimethylpropyl ester & $\mathrm{C}_{7} \mathrm{H}_{12} \mathrm{C}_{12} \mathrm{O}_{2}$ & 198 & 5.02 \\
\hline 39 & 32.98 & Phenyltetrafluorophosphorane & $\mathrm{C}_{6} \mathrm{H}_{5} \mathrm{~F}_{4} \mathrm{P}$ & 184 & 7.52 \\
\hline 40 & 33.12 & 1-Cyclopentyl-2,2-dimethyl-1-propanol & $\mathrm{C}_{10}^{6} \mathrm{H}_{20}^{5} \mathrm{O}$ & 156 & 0.43 \\
\hline 41 & 33.17 & 6-Chloro-2,2,9,9-tetramethyl-3,7-decadiyn5-ol & $\mathrm{C}_{14} \mathrm{H}_{21}^{20} \mathrm{C}_{1 \mathrm{O}}$ & 240 & 1.01 \\
\hline 42 & 33.25 & 3-Methyl-1-[(1H)-1,2,4-triazol-1-yl]butan-2-one & $\mathrm{C}_{7} \mathrm{H}_{11} \mathrm{~N}_{3} \mathrm{O}$ & 153 & 1.26 \\
\hline 43 & 33.30 & Oxalic acid, ethyl neopentyl ester & $\mathrm{C}_{9} \mathrm{H}_{16} \mathrm{O}_{4}$ & 188 & 2.36 \\
\hline 44 & 33.37 & 2,2-Dimethyl-propyl 2,2-dimethyl-propane-thiosulfinate & $\mathrm{C}_{10} \mathrm{H}_{22} \mathrm{OS}_{2}$ & 222 & 4.22 \\
\hline 45 & 33.57 & 2,2-Dimethyl-propyl 2,2-dimethyl-propanesulfinyl sulfone & $\mathrm{C}_{10} \mathrm{H}_{22} \mathrm{O}_{3} \mathrm{~S}_{2}$ & 254 & 1.34 \\
\hline 46 & 33.62 & Oxalic acid, allyl heptyl ester & $\mathrm{C}_{12} \mathrm{H}_{20} \mathrm{O}_{4}$ & 228 & 2.41 \\
\hline 47 & 33.79 & Propane, 1-(2,2-dichloro-1-methylcyclopropyl)-2,2-dimethyl- & $\mathrm{C}_{9} \mathrm{H}_{16} \mathrm{C}_{12}$ & 194 & 5.02 \\
\hline 48 & 33.83 & 2,2-Dimethyl-propyl 2,2-dimethyl-propanesulfinyl sulfone & $\mathrm{C}_{10} \mathrm{H}_{22} \mathrm{O}_{3} \mathrm{~S}_{2}$ & 254 & 0.75 \\
\hline 49 & 33.88 & Dichloroacetic acid, 2,2dimethylpropyl ester & $\mathrm{C}_{7} \mathrm{H}_{12} \mathrm{C}_{12} \mathrm{O}_{2}$ & 198 & 1.41 \\
\hline 50 & 33.95 & Oxalic acid, monoamide, n-propyl, tridecyl ester & $\mathrm{C}_{18} \mathrm{H}_{35} \mathrm{NO}_{3}$ & 313 & 6.73 \\
\hline
\end{tabular}




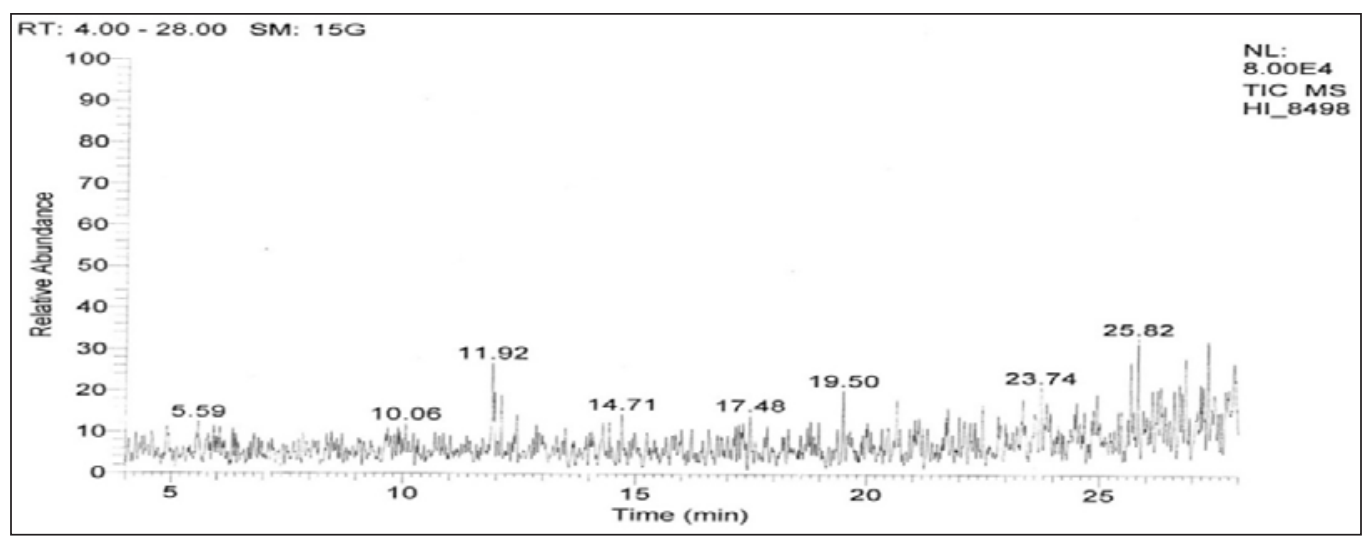

Fig. 2: GC-MS analysis of Triticum species genotype HI 8498

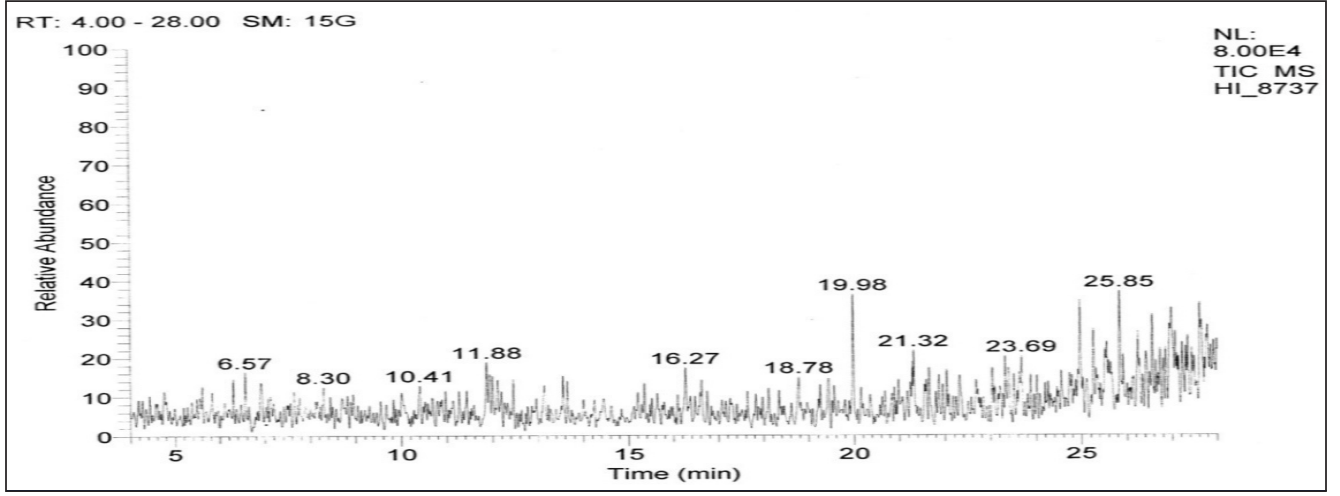

Fig. 3: GC-MS analysis of Triticum species genotype HI 8737

In HI 8498 genotype maximum area $(6.70 \%)$ was observed by Isobutyl pentyl disulfide followed by Tert-Butyl cyclopropylmethyl sulfoxide (6.29\%), Conessine (5.18\%) and 3nPentylthiolane, S, Sdioxide (5.05\%) (Table 4, Fig. 2).

Maximum area (10.48\%) was observed by Cyclohexanemethyl propanoate followed by Isobutyraldehyde, bis (2-methylallyl) acetal $(6.37 \%), 4$-Trifluoroacetoxyoctane $(4.97 \%)$ and 1-Vinylimidazole, 4-nitro-(4.26\%) in HI 8737 genotype (Table 5, Fig. 3).

Compound found with maximum area $(7.14 \%)$ in Dimethylchlorsilyl tert-buthyl peroxide followed by 1,1-Dichloro-1-silacyclohexadiene-2,5 (7.06\%), 1,2,4,5-Tetrazine,3,6-diethyl (6.80\%) and Acetic acid, (2,4,5-trichlorophenoxy)-, isooctyl ester $(6.71 \%)$ in genotype HD 4728 (Table 6, Fig. 4).

Quantification data revealed that the total flavonoid content (free + bound) was observed highest and lowest in durum genotypes of HD $4728(1.75 \mathrm{mg} /$ gdw) and Raj 6560 (0.92 mg/gdw), respectively. But, when compared to the aestivum species, it was noticed that genotypes Raj 4037 (1.38 mg/gdw) and
HI 1544 (0.72 mg/gdw) gave similar trend. In 10 genotypes of 2 wheat species, it was observed that total flavonoids in their bound form was highest in seed of durum genotype HI 8737 (0.63 mg/gdw) and lowest in seed of aestivum genotype Raj 4238 (0.14 $\mathrm{mg} / \mathrm{gdw})$. The total flavonoids content in their free form was highest in durum genotype HD 4728 (1.28 $\mathrm{mg} / \mathrm{gdw}$ ) and lowest in seed of aestivum genotypes HI 1544 (0.39 mg/gdw). Durum genotype HD 4728 gave maximum kaempherol $(1.21 \mathrm{mg} / \mathrm{gdw})$ and quercetin $(0.54 \mathrm{mg} / \mathrm{gdw})$ content and minimum value was given by aestivum genotype HI 1544 (0.58 \& $0.14 \mathrm{mg} / \mathrm{gdw}$ ), respectively (Table 1 ).

Moreover, in plant secondary metabolites occupied chemical and pharmaceutical properties fascinating for health of human being. Presently different kind of compounds belonging to the terpenoids, alkaloids and flavonoids used as medication or as dietary supplements to prevent human from various unknown diseases (Raskin et al. 2002) and in particular a few of these compounds may be efficient for curing various kinds of cancer (Watson et al. 2001; Reddy et al. 2003). 
Table 4: Phytocompounds identified by GC-MS in seed extract of durum wheat genotype (HI 8498)

\begin{tabular}{|c|c|c|c|c|c|}
\hline $\begin{array}{l}\text { Sl. } \\
\text { No. }\end{array}$ & RT & Compound Name & $\begin{array}{c}\text { Molecular } \\
\text { Formula }\end{array}$ & MW & $\begin{array}{c}\text { Area } \\
(\%)\end{array}$ \\
\hline 1 & 11.92 & Phenyltetrafluorophosphorane & $\mathrm{C}_{6} \mathrm{H}_{5} \mathrm{~F}_{4} \mathrm{P}$ & 184 & 3.12 \\
\hline 2 & 11.96 & 2,4-Pentadienoic acid, 1-cyclopenten-3-on-1yl ester & $\mathrm{C}_{10} \mathrm{H}_{10} \mathrm{O}_{3}$ & 178 & 2.28 \\
\hline 3 & 12.11 & 1,3-Benzenediol, O-(2- ethoxyethoxycarbonyl)- & $\mathrm{C}_{10} \mathrm{H}_{12} \mathrm{O}_{5}$ & 212 & 2.17 \\
\hline 4 & 12.44 & Butyric acid, 3amino4methoxy & $\mathrm{C}_{5} \mathrm{H}_{11} \mathrm{NO}_{2} \mathrm{~S}$ & 149 & 1.06 \\
\hline 5 & 14.44 & 20-Carboethoxy-20-demethylvincadifformine & $\mathrm{C}_{23} \mathrm{H}_{28} \mathrm{~N}_{2} \mathrm{O}_{4}$ & 396 & 1.10 \\
\hline 6 & 17.48 & 1-Benzylcyclopentanol-1 & $\mathrm{C}_{12} \mathrm{H}_{16} \mathrm{O}$ & 176 & 0.94 \\
\hline 7 & 18.80 & 1,2,3-Oxadiazol-3-ium, 5-trifluoroacetylamidato-3-methyl & $\mathrm{C}_{5} \mathrm{H}_{4} \mathrm{~F}_{3} \mathrm{~N}_{3} \mathrm{O}_{2}$ & 195 & 0.80 \\
\hline 8 & 19.50 & $\begin{array}{l}\text { Cholestan-6-one, 3-(2-hydroxypropoxy)-cyclic1,2-propanediyl } \\
\text { acetal, (3á,5à)- }\end{array}$ & $\mathrm{C}_{33} \mathrm{H}_{58} \mathrm{O}_{4}$ & 518 & 2.40 \\
\hline 9 & 20.66 & 4Butylbenzoic acid, octadecyl ester & $\mathrm{C}_{27} \mathrm{H}_{46} \mathrm{O}_{2}$ & 402 & 1.39 \\
\hline 10 & 21.05 & Benzene, tert-butyl- & $\mathrm{C}_{10} \mathrm{H}_{14}$ & 134 & 0.80 \\
\hline 11 & 21.14 & Octane, 1,1'-[ethylidenebis(oxy)]bis- & $\mathrm{C}_{18} \mathrm{H}_{38} \mathrm{O}_{2}$ & 286 & 0.84 \\
\hline 12 & 21.22 & Hydroxyurea, N,N,N',O-tetramethyl- & $\mathrm{C}_{5} \mathrm{H}_{12} \mathrm{~N}_{2} \mathrm{O}_{2}$ & 132 & 0.84 \\
\hline 13 & 21.71 & Butyric acid, crotyl ester & $\mathrm{C}_{8} \mathrm{H}_{14} \mathrm{O}_{2}$ & 142 & 0.82 \\
\hline 14 & 21.74 & 3Ethoxycarbonylquinoxaline 1oxide & $\mathrm{C}_{11} \mathrm{H}_{10} \mathrm{~N}_{2} \mathrm{O}_{3}$ & 218 & 1.53 \\
\hline 15 & 21.99 & Succinic acid, 4methoxyphenyl nonyl ester & $\mathrm{C}_{20} \mathrm{H}_{30} \mathrm{O}_{5}$ & 350 & 1.13 \\
\hline 16 & 22.33 & Azobenzene & $\mathrm{C}_{12} \mathrm{H}_{10} \mathrm{~N}_{2}$ & 182 & 0.94 \\
\hline 17 & 22.49 & Benzenesulfonamide, N,4dimethyl & $\mathrm{C}_{8} \mathrm{H}_{11} \mathrm{NO}_{2} \mathrm{~S}$ & 185 & 1.49 \\
\hline 18 & 22.82 & 6Uracilsulfonamide & $\mathrm{C}_{4} \mathrm{H}_{5} \mathrm{~N}_{3} \mathrm{O}_{4} \mathrm{~S}$ & 191 & 1.09 \\
\hline 19 & 22.98 & Benzenepentanamine & $\mathrm{C}_{11} \mathrm{H}_{17} \mathrm{~N}$ & 163 & 0.97 \\
\hline 20 & 23.36 & 5-Chlorovaleramide, N-(2-fluorophenyl)- & $\mathrm{C}_{11} \mathrm{H}_{13} \mathrm{ClFNO}$ & 229 & 1.74 \\
\hline 21 & 23.73 & 1,3-Benzenediol, o-acryloyl-o'-heptafluorobutyryl- & $\mathrm{C}_{13} \mathrm{H}_{7} \mathrm{~F}_{7} \mathrm{O}_{4}$ & 360 & 4.40 \\
\hline 22 & 23.86 & 2,4-Dihydroxy-2,5-dimethyl-3(2H)-furan-3-one & $\mathrm{C}_{6} \mathrm{H}_{8} \mathrm{O}_{4}$ & 144 & 1.13 \\
\hline 23 & 24.50 & Benzenemethanol, 2-chloro-à-[[(1-methylethyl)amino]methyl]- & $\mathrm{C}_{11} \mathrm{H}_{16} \mathrm{ClNO}$ & 213 & 1.53 \\
\hline 24 & 24.67 & 4-Spirohexanone-5,5-dichloro- & $\mathrm{C}_{6} \mathrm{H}_{6} \mathrm{C}_{12} \mathrm{O}$ & 164 & 1.11 \\
\hline 25 & 24.87 & 2H-Pyran,2-(butylthio)tetrahydro- & $\mathrm{C}_{9} \mathrm{H}_{18} \mathrm{OS}$ & 174 & 0.88 \\
\hline 26 & 24.94 & Propanamide, N-(1-naphthyl)-2,2-dimethyl- & $\mathrm{C}_{15} \mathrm{H}_{17} \mathrm{NO}$ & 227 & 1.76 \\
\hline 27 & 25.41 & Trifluoromethyl t-butyl sulfide & $\mathrm{C}_{5} \mathrm{H}_{9} \mathrm{~F}_{3} \mathrm{~S}$ & 158 & 1.23 \\
\hline 28 & 25.46 & Imidazole, 5tbuyloxycarbonylamino4fluoro & $\mathrm{C}_{8} \mathrm{H}_{12} \mathrm{FN}_{3} \mathrm{O}_{2}$ & 201 & 2.00 \\
\hline 29 & 25.61 & Acrylic acid, 2,4dichloronaphthyllester & $\mathrm{C}_{13} \mathrm{H}_{8} \mathrm{C}_{12} \mathrm{O}_{2}$ & 266 & 0.98 \\
\hline 30 & 25.67 & Decane, 2,2,9trimethyl & $\mathrm{C}_{13} \mathrm{H}_{28}$ & 184 & 3.76 \\
\hline 31 & 25.73 & $\begin{array}{l}\text { Fumaric acid, monoamide, N,N-dimethyl-, 4-isopropoxyphenyl } \\
\text { ester }\end{array}$ & $\mathrm{C}_{15} \mathrm{H}_{19} \mathrm{NO}_{4}$ & 277 & 0.96 \\
\hline 32 & 25.82 & 3nPentylthiolane, S,Sdioxide & $\mathrm{C}_{9} \mathrm{H}_{18} \mathrm{O}_{2} \mathrm{~S}$ & 190 & 5.05 \\
\hline 33 & 25.94 & Oxalic acid, ethyl isobutyl ester & $\mathrm{C}_{8} \mathrm{H}_{14} \mathrm{O}_{4}$ & 174 & 0.84 \\
\hline 34 & 26.13 & Butanoic acid, 2-oxo & $\mathrm{C}_{4} \mathrm{H}_{6} \mathrm{O}_{3}$ & 102 & 2.55 \\
\hline 35 & 26.24 & 1,2,4Benzenetriol, triacetate & $\mathrm{C}_{12} \mathrm{H}_{12} \mathrm{O}_{6}$ & 252 & 1.74 \\
\hline 36 & 26.31 & 2,3Dimethyl-5-(2,6,10-trimethylundecyl) thiophene & $\mathrm{C}_{20} \mathrm{H}_{36} \mathrm{~S}$ & 308 & 1.76 \\
\hline 37 & 26.41 & 2-Furancarboxylic acid, 3,5-difluorophenyl ester & $\mathrm{C}_{11} \mathrm{H}_{6} \mathrm{~F}_{2} \mathrm{O}_{3}$ & 224 & 0.82 \\
\hline 38 & 26.61 & Carbonic acid, bis(2-methylpropyl) ester & $\mathrm{C}_{9} \mathrm{H}_{18} \mathrm{O}_{3}$ & 174 & 3.03 \\
\hline 39 & 26.72 & 3-Methylbut-2-enoic acid, 2-diethylaminoethyl ester & $\mathrm{C}_{11} \mathrm{H}_{21} \mathrm{NO}_{2}$ & 199 & 2.65 \\
\hline 40 & 26.78 & 2,7-Dimethyl-2,6-octadien-4-ol & $\mathrm{C}_{10} \mathrm{H}_{18} \mathrm{O}$ & 154 & 1.92 \\
\hline 41 & 26.85 & Conessine & $\mathrm{C}_{24} \mathrm{H}_{40} \mathrm{~N}_{2}$ & 356 & 5.18 \\
\hline
\end{tabular}




\begin{tabular}{cclccc}
42 & 26.93 & 1,2Cyclohexanedimethanol & $\mathrm{C}_{8} \mathrm{H}_{16} \mathrm{O}_{2}$ & 144 & 0.86 \\
43 & 27.17 & $\begin{array}{l}\text { 6-Thiopyrazolo[3,4-d]pyrimidin-4,6(5H,7H)-dione-3-carbo } \\
\text { xamide }\end{array}$ & $\mathrm{C}_{6} \mathrm{H}_{5} \mathrm{~N}_{5} \mathrm{O}_{2} \mathrm{~S}$ & 211 & 2.11 \\
44 & 27.23 & 4-Ethenylhexadecyloxybenzene & $\mathrm{C}_{24} \mathrm{H}_{40} \mathrm{O}$ & 344 & 2.19 \\
45 & 27.33 & Tert-Butyl cyclopropylmethyl sulfoxide & $\mathrm{C}_{8} \mathrm{H}_{16} \mathrm{O}$ & 160 & 6.29 \\
46 & 27.47 & Urea, N,N'-di-2-propenyl- & $\mathrm{C}_{7} \mathrm{H}_{12} \mathrm{~N}_{2} \mathrm{O}$ & 140 & 2.69 \\
47 & 27.63 & 2Ethylbutyl propionate & $\mathrm{C}_{9} \mathrm{H}_{18} \mathrm{O}_{2}$ & 158 & 1.12 \\
48 & 27.72 & 2-Butene, 1-bromo- & $\mathrm{C}_{4} \mathrm{H}_{7} \mathrm{Br}_{2}$ & 134 & 1.97 \\
49 & 27.79 & $\begin{array}{l}\text { Propanoic acid, 2,2-dimethyl-, 2-(1,1-dimethylethyl)-4- } \\
\text { methylphenyl ester }\end{array}$ & $\mathrm{C}_{16} \mathrm{H}_{24} \mathrm{O}_{2}$ & 248 & 3.36 \\
50 & 27.91 & Isobutyl pentyl disulfide & $\mathrm{C}_{9} \mathrm{H}_{20} \mathrm{~S}_{2}$ & 192 & 6.70 \\
\hline
\end{tabular}

Table 5: Phytocompounds identified by GC-MS in seed extract of durum wheat genotype (HI 8737)

\begin{tabular}{|c|c|c|c|c|c|}
\hline $\begin{array}{l}\text { Sl. } \\
\text { No. }\end{array}$ & RT & Compound Name & $\begin{array}{c}\text { Molecular } \\
\text { Formula } \\
\end{array}$ & MW & $\begin{array}{c}\text { Area } \\
(\%)\end{array}$ \\
\hline 1 & 5.61 & Isonipecotic acid, $\mathrm{N}$-(4-butylbenzoyl)-, butyl ester & $\mathrm{C}_{21} \mathrm{H}_{31} \mathrm{NO}_{3}$ & 345 & 0.58 \\
\hline 2 & 6.30 & $\begin{array}{l}\text { 1H-Pyrrolizine-1-methanol, hexahydro-7-hydroxy, } \\
\text { [1S-(1à,7à,7aá)]- }\end{array}$ & $\mathrm{C}_{8} \mathrm{H}_{15} \mathrm{NO}_{2}$ & 157 & 0.89 \\
\hline 3 & 6.57 & 1,2-Ethanediamine, N,N-dimethyl- & $\mathrm{C}_{4} \mathrm{H}_{12} \mathrm{~N}_{2}$ & 88 & 1.34 \\
\hline 4 & 11.88 & $\begin{array}{l}\text { 6-Ethoxycarbonyl-7-oxo-1,7-dihydro-[1,2,4]triazolo(4,3-b)[ } \\
\text { 1,2,4]-triazine }\end{array}$ & $\mathrm{C}_{7} \mathrm{H}_{7} \mathrm{~N}_{5} \mathrm{O}_{3}$ & 209 & 2.02 \\
\hline 5 & 11.94 & Furfuryl sulfide & $\mathrm{C}_{10} \mathrm{H}_{10} \mathrm{O}_{2} \mathrm{~S}$ & 194 & 2.07 \\
\hline 6 & 12.00 & Oxalic acid, cyclohexylmethyl undecyl ester & $\mathrm{C}_{20} \mathrm{H}_{36} \mathrm{O}_{4}$ & 340 & 1.08 \\
\hline 7 & 12.46 & 5-Pyrimidinol, 2-methyl- & $\mathrm{C}_{5} \mathrm{H}_{6} \mathrm{~N}_{2} \mathrm{O}$ & 110 & 1.11 \\
\hline 8 & 13.55 & But-3-enyl (E)-2-methylbut-2-enoate & $\mathrm{C}_{9} \mathrm{H}_{14} \mathrm{O}_{2}$ & 154 & 0.97 \\
\hline 9 & 13.64 & Thiophen-2-methylamine, N,N-diheptyl- & $\mathrm{C}_{19} \mathrm{H}_{35} \mathrm{NS}$ & 309 & 0.99 \\
\hline 10 & 16.27 & $\begin{array}{l}\text { Trifluoromethyl tbutyl } \\
\text { disulfide }\end{array}$ & $\mathrm{C}_{5} \mathrm{H}_{9} \mathrm{~F}_{3} \mathrm{~S}_{2}$ & 190 & 1.31 \\
\hline 11 & 16.62 & Isophthalic acid, 2-fluorophenyl isobutyl ester & $\mathrm{C}_{18} \mathrm{H}_{17} \mathrm{FO}_{4}$ & 316 & 0.76 \\
\hline 12 & 18.78 & 1,1Dimethoxypent4en3one & $\mathrm{C}_{7} \mathrm{H}_{12} \mathrm{O}_{3}$ & 144 & 0.74 \\
\hline 13 & 19.98 & Sedoheptulosan tetrabenzoate & $\mathrm{C}_{35} \mathrm{H}_{28} \mathrm{O}_{10}$ & 608 & 3.70 \\
\hline 14 & 20.89 & Fumaric monoamide, N-(2-bromophenyl)-, nonyl ester & $\mathrm{C}_{19} \mathrm{H}_{26} \mathrm{BrNO}_{3}$ & 395 & 0.79 \\
\hline 15 & 21.32 & 2,6-Octadiene-4,5-diol, 3,6-dimethyl- & $\mathrm{C}_{10} \mathrm{H}_{18} \mathrm{O}_{2}$ & 170 & 1.79 \\
\hline 16 & 21.66 & 1,1,1,3,3-Pentafluoroacetone, fluoroimine & $\mathrm{C}_{3} \mathrm{HF}_{6} \mathrm{~N}$ & 165 & 1.46 \\
\hline 17 & 22.04 & Silane, 2-butenyltrichloro-, (Z)- & $\mathrm{C}_{4} \mathrm{H}_{7} \mathrm{Cl}_{3} \mathrm{Si}$ & 188 & 1.25 \\
\hline 18 & 23.04 & Propanal, butylhydrazone & $\mathrm{C}_{7} \mathrm{H}_{16} \mathrm{~N}_{2}$ & 128 & 1.51 \\
\hline 19 & 23.32 & $\begin{array}{l}\text { Chromium,[(1,2,3,4ü)-1,3-cycloheptadiene]( ü7- } \\
\text { cycloheptatrienylium)- }\end{array}$ & $\mathrm{C}_{14} \mathrm{H}_{17} \mathrm{Cr}$ & 237 & 1.63 \\
\hline 20 & 23.39 & Thiepane, 1,1dioxide & $\mathrm{C}_{6} \mathrm{H}_{12} \mathrm{O}_{2} \mathrm{~S}$ & 148 & 1.97 \\
\hline 21 & 23.52 & Sydnone, 3(1,1-dimethylethyl)- & $\mathrm{C}_{6} \mathrm{H}_{10} \mathrm{~N}_{2} \mathrm{O}_{2}$ & 142 & 1.65 \\
\hline 22 & 23.69 & Benzenesulfonic acid, methyl ester & $\mathrm{C}_{7} \mathrm{H}_{8} \mathrm{O}_{3} \mathrm{~S}$ & 172 & 1.68 \\
\hline 23 & 23.88 & 2,5Difluorobenzoic acid, 4cyanophenyl ester & $\mathrm{C}_{14} \mathrm{H}_{7} \mathrm{~F}_{2} \mathrm{NO}_{2}$ & 259 & 1.02 \\
\hline 24 & 24.02 & 2,4(3H,5H)-Furandione, 3,5-dimethyl- & $\mathrm{C}_{6} \mathrm{H}_{8} \mathrm{O}_{3}$ & 128 & 0.83 \\
\hline 25 & 24.55 & Trifluoromethyldifluorophosphine & $\mathrm{CF}_{5} \mathrm{P}$ & 138 & 0.70 \\
\hline 26 & 24.97 & 1-Vinylimidazole, 4-nitro- & $\mathrm{C}_{5} \mathrm{H}_{5} \mathrm{~N}_{3} \mathrm{O}_{2}$ & 139 & 4.26 \\
\hline 27 & 25.26 & 4-(Iodomethyl)-1-azabicyclo[2.2.2]octane & $\mathrm{C}_{8} \mathrm{H}_{14} \mathrm{IN}$ & 251 & 2.71 \\
\hline 28 & 25.52 & Undecane, 6-methyl- & $\mathrm{C}_{12} \mathrm{H}_{26}$ & 170 & 1.56 \\
\hline 29 & 25.56 & 3-Buten-1-ol, propanoate & $\mathrm{C}_{7} \mathrm{H}_{12} \mathrm{O}_{2}$ & 128 & 2.48 \\
\hline 30 & 25.85 & 4-Trifluoroacetoxyoctane & $\mathrm{C}_{10} \mathrm{H}_{17} \mathrm{~F}_{3} \mathrm{O}_{2}$ & 226 & 4.97 \\
\hline
\end{tabular}




\begin{tabular}{|c|c|c|c|c|c|}
\hline 31 & 25.92 & 2,6Octadiene & $\mathrm{C}_{8} \mathrm{H}_{14}$ & 110 & 1.24 \\
\hline 32 & 26.25 & 2,2-Dimethylnon-5-en-3-one & $\mathrm{C}_{11} \mathrm{H}_{20} \mathrm{O}$ & 168 & 3.08 \\
\hline 33 & 26.42 & Spiropentane & $\mathrm{C}_{5} \mathrm{H}_{8}$ & 68 & 3.74 \\
\hline 34 & 26.50 & 4-(2-Dimethylaminoethoxy) benzonitrile & $\mathrm{C}_{11} \mathrm{H}_{14} \mathrm{~N}_{2} \mathrm{O}$ & 190 & 1.40 \\
\hline 35 & 26.56 & 4Octene, 2,3,6,7tetramethyl & $\mathrm{C}_{12} \mathrm{H}_{24}$ & 168 & 3.27 \\
\hline 36 & 26.64 & Oxalic acid, neopentyl propyl ester & $\mathrm{C}_{10} \mathrm{H}_{18} \mathrm{O}_{4}$ & 202 & 1.34 \\
\hline 37 & 26.73 & 1,3Benzodioxole, 2hexyloxy & $\mathrm{C}_{13} \mathrm{H}_{18} \mathrm{O}_{3}$ & 222 & 1.60 \\
\hline 38 & 26.81 & Butanimidamide, N-(1-chloro-2-methyl-1-butenyl)-2-methyl- & $\mathrm{C}_{10} \mathrm{H}_{19} \mathrm{ClN}_{2}$ & 202 & 1.50 \\
\hline 39 & 26.86 & 3-Iodopropanesulfonic acid, methyl ester & $\mathrm{C}_{4} \mathrm{H}_{9} \mathrm{IO}_{3} \mathrm{~S}$ & 264 & 1.46 \\
\hline 40 & 26.99 & Cyclohexanemethyl propanoate & $\mathrm{C}_{10} \mathrm{H}_{18} \mathrm{O}_{2}$ & 170 & 10.48 \\
\hline 41 & 27.07 & 2-Furancarboxylic acid, 2-formyl-4,6-dichlorophenyl ester & $\mathrm{C}_{12} \mathrm{H}_{6} \mathrm{C}_{12} \mathrm{O}_{4}$ & 284 & 1.75 \\
\hline 42 & 27.22 & 1,3-Dinitro-2-imidazolidinone & $\mathrm{C}_{3} \mathrm{H}_{4} \mathrm{~N}_{4} \mathrm{O}_{5}$ & 176 & 1.46 \\
\hline 43 & 27.30 & 2-Butanone,1-(2-furanyl)-3methyl- & $\mathrm{C}_{9} \mathrm{H}_{12} \mathrm{O}_{2}$ & 152 & 1.24 \\
\hline 44 & 27.35 & Propargyl alcohol, trifluoroacetate & $\mathrm{C}_{5} \mathrm{H}_{3} \mathrm{~F}_{3} \mathrm{O}_{2}$ & 152 & 1.61 \\
\hline 45 & 27.44 & 4Nonene,2,3,3trimethyl,(E) & $\mathrm{C}_{12} \mathrm{H}_{24}$ & 168 & 0.94 \\
\hline 46 & 27.61 & Isobutyraldehyde, bis(2-methylallyl) acetal & $\mathrm{C}_{12} \mathrm{H}_{22} \mathrm{O}_{2}$ & 198 & 6.37 \\
\hline 47 & 27.78 & 1,3-Benzenediol, O-cyclopropanecarbonyl-O'-pivaloyl- & $\mathrm{C}_{15} \mathrm{H}_{18} \mathrm{O}_{4}$ & 262 & 4.00 \\
\hline 48 & 27.86 & Butanimidamide, N-(1-chloro-2-methyl-1-butenyl)-2-methyl- & $\mathrm{C}_{10} \mathrm{H}_{19} \mathrm{ClN}_{2}$ & 202 & 0.92 \\
\hline 49 & 27.92 & Bicyclo[3.2.1]octane2,3,4trione & $\mathrm{C}_{8} \mathrm{H}_{8} \mathrm{O}_{3}$ & 152 & 1.10 \\
\hline 50 & 28.00 & (CH3)2NCH2Si(CH3)3 & $\mathrm{C}_{6} \mathrm{H}_{17} \mathrm{NSi}$ & 131 & 1.63 \\
\hline
\end{tabular}

Table 6: Phytocompounds identified by GC-MS in seed extract of durum wheat genotype HD 4728

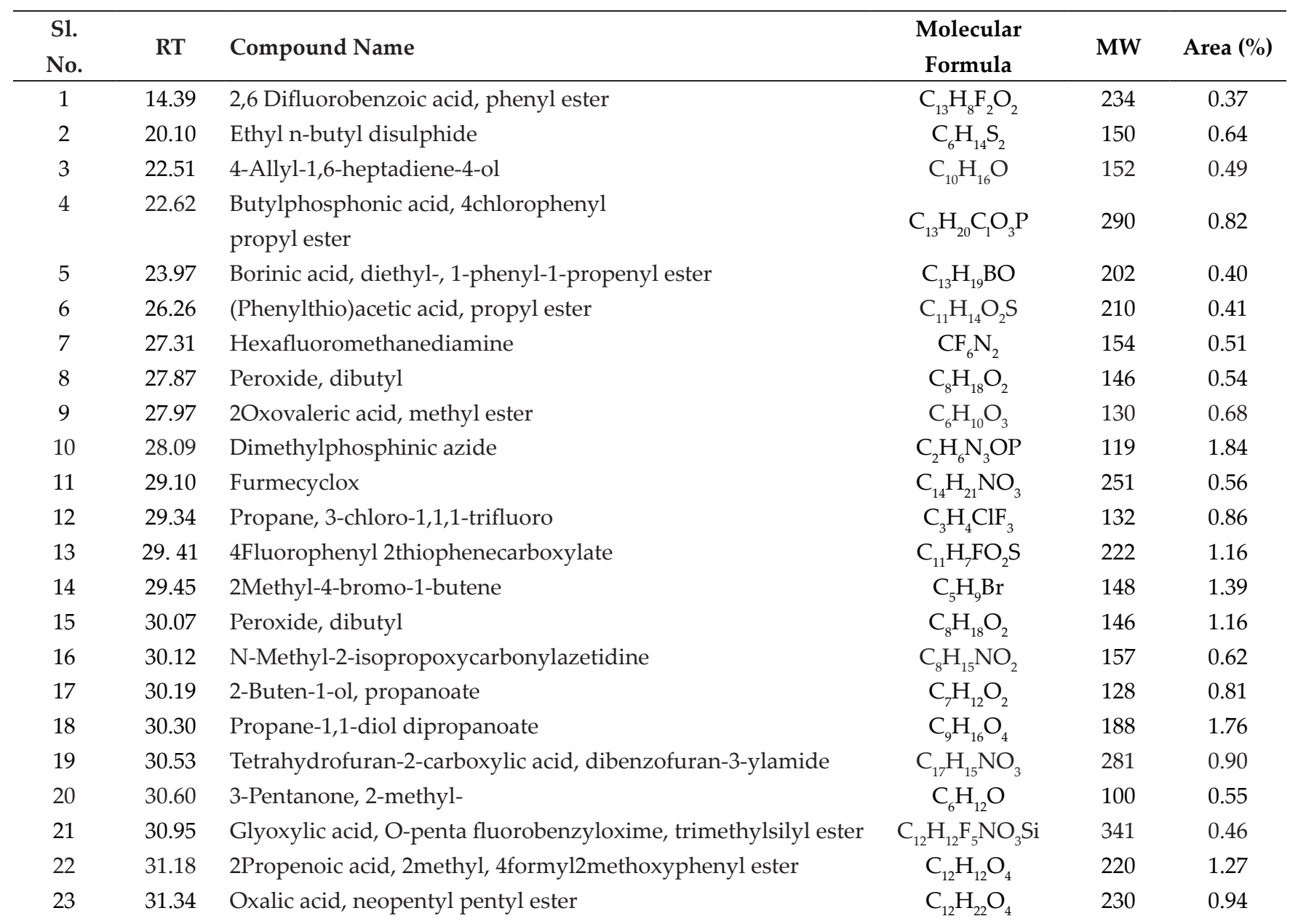


24

31.43 2-(1-Methylcyclohexyloxy)-tetrahydropyran

$\begin{array}{ccc}\mathrm{C}_{12} \mathrm{H}_{22} \mathrm{O}_{2} & 198 & 1.40 \\ \mathrm{C}_{11} \mathrm{H}_{12} \mathrm{Br}_{3} \mathrm{NO} & 411 & 1.55\end{array}$

25

31.57 Propanamide, 2,2-dimethyl-N (2,4,6-tribromophenyl)-

366

1.40

31.71 Methane, bis[4-(2,2dimethylpropanamido) phenyl]-

31.79 Butanoic acid, heptafluoro-, 1-(butoxycarbonyl)propyl ester

31.85 Benzenamine, 4chloro-N-[(4nitrophenyl) methylene]-,N-oxide

$\mathrm{C}_{12} \mathrm{H}_{15} \mathrm{~F}_{7} \mathrm{O}_{4} \quad 356$

3.25

$\mathrm{C}_{13} \mathrm{H}_{9} \mathrm{ClN}_{2} \mathrm{O}_{3}$

276

0.58

31.89 Benzaldehyde, 3-benzyloxy-2-fluoro-

$\mathrm{C}_{14} \mathrm{H}_{11} \mathrm{FO}_{2}$

230

1.09

31.96 4-Hydroxybutyl acrylate

32.08 2-Pentanone, 1(2-furanyl)-

32.12 2,2-Dimethylpropanoic acid, cyclobutyl ester

$\mathrm{C}_{7} \mathrm{H}_{12} \mathrm{O}_{3}$

144

0.54

$\mathrm{C}_{9} \mathrm{H}_{12} \mathrm{O}_{2}$

152

2.02

$\mathrm{C}_{9} \mathrm{H}_{16} \mathrm{O}_{2}$

156

2.82

32.28

4,8-Dioxatricyclo[5.1.0.0(3,5)]octane, 1-methyl-5-(1methylethyl)-,(1à,3á,5á,7à)-

32.46

2-Ethylthiolane, S,S-dioxide

$\mathrm{C}_{10} \mathrm{H}_{16} \mathrm{O}_{2}$

168

6.30

$\mathrm{C}_{6} \mathrm{H}_{12} \mathrm{O}_{2} \mathrm{~S}$

148

1.19

$\mathrm{C}_{10} \mathrm{H}_{22} \mathrm{OS}_{2}$

222

1.12

32.603 3-Butenoic acid, ethyl ester

32.68 1-Phenyl-1-nonanol

$\mathrm{C}_{6} \mathrm{H}_{10} \mathrm{O}_{2}$

1.67

$\mathrm{C}_{15} \mathrm{H}_{24} \mathrm{O}$

114

5.59

$\mathrm{C}_{11} \mathrm{H}_{24}$

220

3.38

32.88

Octane, 2,3,6-trimethy-1-

$\mathrm{C}_{9} \mathrm{H}_{8} \mathrm{O}_{3}$

156

2.20

33.01 Oxalic acid, isobutyl propyl ester

33.07 Acetic acid, (2,4,5-trichlorophenoxy)-, isooctyl ester

$\mathrm{C}_{9} \mathrm{H}_{16} \mathrm{O}_{4}$

164

2.38

$\mathrm{C}_{16} \mathrm{H}_{21} \mathrm{Cl}_{3} \mathrm{O}_{3}$

188

6.71

33.14

cis2,4Dimethylthiane, S,Sdioxide

$\mathrm{C}_{7} \mathrm{H}_{14} \mathrm{O}_{2} \mathrm{~S}$

366

2.24

33.26 Chloroacetic acid, 2,2-dimethylpropyl ester

$\mathrm{C}_{7} \mathrm{H}_{13} \mathrm{ClO}_{2}$

162

1.24

33.37 1,3,7-Nonatriene-1,1-dicarbonitrile, 4,8-dimethyl-, (E)-

33.46 1,2:3,4-Bis-O-isopropylidene-d-galactopyranose sulfamate

$\mathrm{C}_{13} \mathrm{H}_{16} \mathrm{~N}$

164

2.80

$\mathrm{C}_{12} \mathrm{H}_{21} \mathrm{NO}_{8} \mathrm{~S} \quad 339$

3.23

33.57

Dimethylchlorsilyl tert-buthyl peroxide

$\mathrm{C}_{6} \mathrm{H}_{15} \mathrm{ClO}_{2} \mathrm{Si}$

7.14

33.66

Cis-2-Nitro-4-t-butylcyclohexanone

$\mathrm{C}_{10} \mathrm{H}_{17} \mathrm{NO}_{3}$

2.50

33.74 1,2,4,5-Tetrazine,3,6-diethyl-

33.85 Oxalic acid, cyclobutyl isohexyl ester

33.98 1,1-Dichloro-1-silacyclohexadiene-2,5

$\mathrm{C}_{6} \mathrm{H}_{10} \mathrm{~N}_{4}$

6.80

$\mathrm{C}_{12} \mathrm{H}_{20} \mathrm{O}_{4}$

2.67

$\mathrm{C}_{5} \mathrm{H}_{6} \mathrm{Cl}_{2} \mathrm{Si}$

7.06

RT-Retantion Time, MW-Molecular weight.

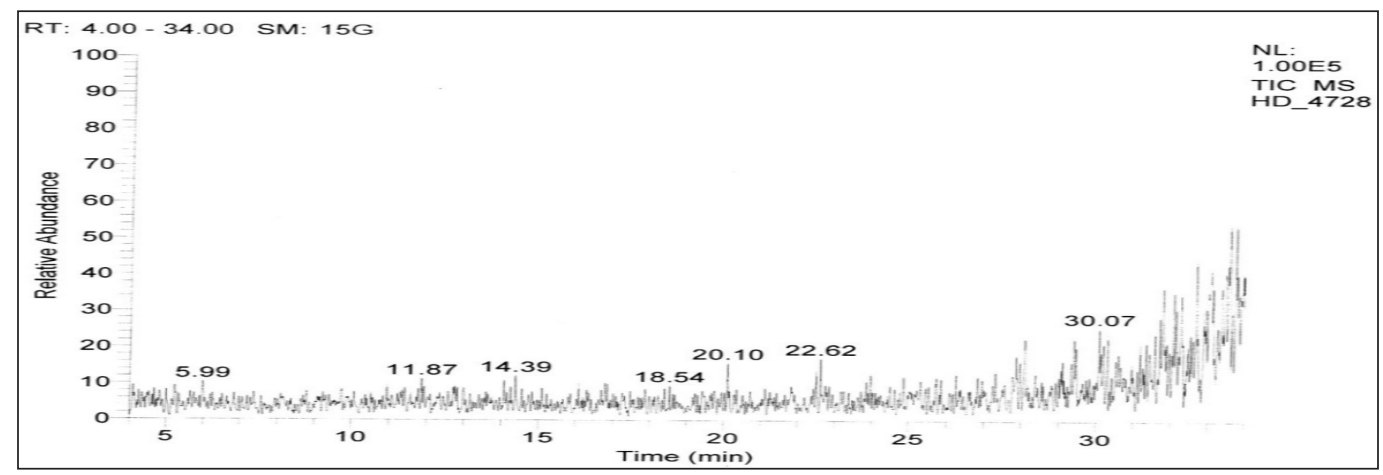

Fig. 4: GC-MS analysis of Triticum species genotype HD 4728

In the present investigation, flavonoid has been extracted from seed of Triticum $s p$. The isolated compounds were identified and confirmed by using the techniques like chromatography and spectroscopy. In various kinds of phytonutrients, flavonoids are the strongest antioxidant with having different helpful properties i.e. anti inflammatory and immunity. Flavonoids rich diets are associated with cancer and cardio vascular disease prevention. Although, this is not yet much determined that flavonoid are main factor for this. Flavonoids are a part of polyphenol class of phytonutrients. There are various significant sub-groups of flavonoids. Some important are including anthocynidins, flavanols, flavones, flavonols, flavonones and isoflavones. It is consumed to reduce the risk of heart related 

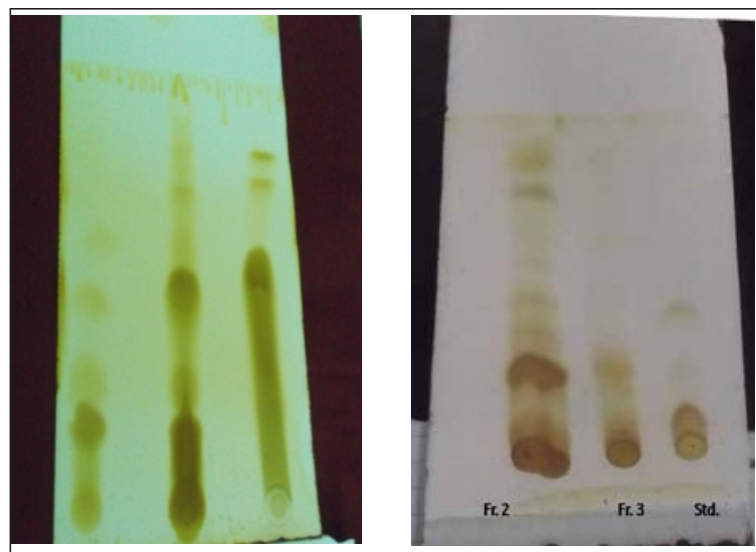

MPO 1215
HI 8498

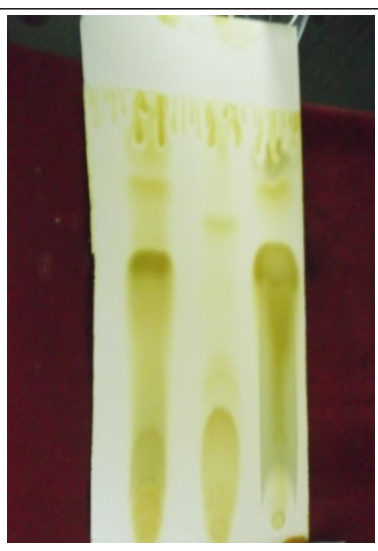

HI 8737

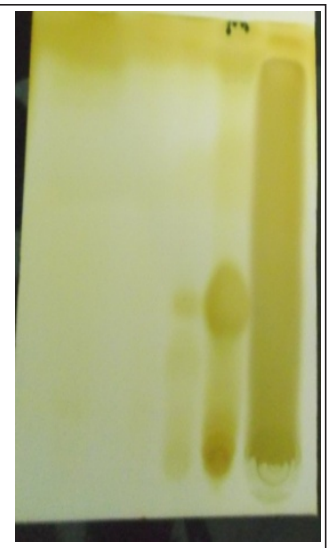

HD 4728

Fig. 5: TLC plates of Triticum genotypes

diseases in the body (Urquiaga and Leighton 2000). As anti-cancer activity treatment, they inhibit the initiation, promotion and progression of the several kinds of tumors (Urquiaga and Leighton 2000; Okwu 2004). Flavonoid compounds seem to have significant characters to stop against pathogens, predators and contribute to physiological activities like seed maturation and dormancy (Winkel-Shirley 2002).

\section{ACKNOWLEDGEMENTS}

Authors gratefully acknowledge scientist and research staff of Seminal Applied Science laboratory, Jaipur (Raj.) for providing related material/ equipment and Technical support.

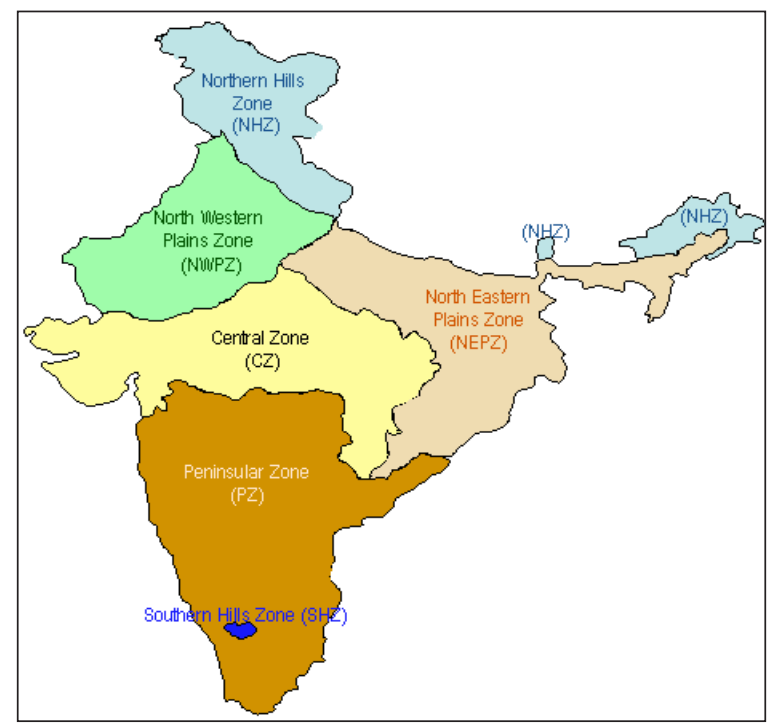

Map I: Wheat growing mega zones of India

Source: http://www.krishisewa.com/agroclimatic-zones/wheatgrowing-zones.html

\section{REFERENCES}

Anonymous, 2014. http://www.theguardian.com/ globaldevelopment-professionals- network / 2014/ apr/ 01/international-wheat-yield- partnership-food-security.

Brenda W.S. 1998. Flavonoids in seeds and grains. Physiological function, agronomic importance and the genetics of biosynthesis. Seed Science Research, 8: 415-422.

Calderon Montano J.M., Burgos Moron E., Perez Guerrero C. and Lopez Lazaro M. 2011. "A Review on the dietary flavonoid kaempferol". Mini Review of Medicinal Chemistry, 11(4): 298-344.

Dykes L. and Rooney W. 2007. Phenolic compounds in cereal grains and their health benefits. Cereal Foods World, 52: 105-111.

Formica, J.V. and Regelson W. 1995. "Review of the biology of Quercetin and related bioflavonoids". Food and Chemical Toxicology, 33(12): 1061-80.

Garcia, Mediavilla, V., Crespo, I., Collado, P.S., Esteller, A., Sanchez Campos, S., Tunon, M.J. and Gonzalez Gallego, J. 2007. The anti-inflammatory flavones quercetin and kaempferol cause inhibition of inducible nitric oxide synthase, cyclooxygenase $-2^{\text {nd }}$ reactive $C$ proteins, and down regulation of the nuclear factor kappa $b$ pathway in chang liver cells. European Journal of Pharmacology, 557: 221-229.

Harborne, J.B. 1973. Phytochemical Methods: A Guide to Modern Techniques of plant Analysis. Chapman and Hall Ltd, London, pp. 279.

Kim, J.Y., Parkm S.J., Yunm K.J., Cho, Y.W., Park, H.J. and Lee, K.T. 2008. Isoliquiritigenin isolated from the roots of Glycyrrhiza uralensis inhibits LPS iduced NOS and COX-2 expression via the attenuation of NF kappa B in RAW 264.7 macrophages. European Journal of Pharmacology, 584: 175-184.

Kumar, J., Singh, S.K., Singh, L., Kumar, A., Anurag Singh, S.K. and Kumar, M. 2016. Estimates of general and specific combining ability for grain yield and other physiological 
characters in bread wheat under late sown condition. Research in Environment and Life Science, 9(7): 784-789.

MacIntosh, C.J. 2008. Wheatgrass and mold. Available from: http:// www.cityfarmer.org/wheatgrass.html. [Last accessed on Jun 10].

Middleton, E. and Kandasuami, C. 1993. The impact of plant flavonoids on mammalian biology: Implications for immunity, inflammation and cancer, in the flavonoids. Advances in Research Science (Ed.) Harbone, I.R., Chapman and Hall, London. 619-645.

Mohammadi Joo, S., Mirasi, A., Saeidi aboeshaghi, R. and Amiri, M. 2015. Evaluation of bread wheat (Triticum aestivum $L$.) genotypes based on resistance indices under field conditions. International Journal of Biosciences, 6(2): 331-337.

Okwu, D.E. 2004. Phytochemicals and vitamin content of indigenous spices of South Eastern. Nigeria Journal of Sustainable Agriculture and Environment, 6: 30-34.

Pandey, M.K., Sandur, S.K., Sung, B., Sethi, G., Kunnumakkara, A.B. and Agarwal, B.B. 2007. Butein, a tetrahydroxychalcone, inhibits nuclear factor (NF) kappa B and NF - Kappa B regulated gene expression trhough direct inhibition of I Kappa B alpha kinase beta on cysteine $17 \mathrm{~g}$ residue. Journal of Biological Chemistry, 282: $17340-17350$.

Pichersky, E. and Gang, D.R. 2000. Genetics and Biochemistry of Secondary Metabolites in Plants: An Evolutionary Perspective. Trends in Plant Science Perspectives, 5: 439-445.

Peer, W.A. and Murphy, A.S. 2007. Flavonoids and auxin transport : modulators are regulators?. Trends in Plant Science, 12: 556-563.

Raskin, I., Ribnicky, D.M., Komarnytsky, S., Ilic, N., Poulev, A., Borisjuk, N., Brinker, A., Moreno, D.A., Ripoll, C. and Yakoby, N. 2002. Plants and human health in the twentyfirst century. Trends in Biotechnology, 20: 522-531.
Reddy, L., Odhav, B. and Bhoola, K.D. 2003. Natural products for cancer prevention: a global perspective. Pharmacology \& Therapeutics, 99: 1-13.

Sharma, P. and Sarin, R. 2012c. Isolation and characterization of quercetin and kaempferol in vivo and in vitro from Pedalium murex. International Research Journal of Pharmacy, 3(6): 184-187.

Sharma, P. and Sarin, R. 2012d. Isolation and identification of flavonoids from Sesamum indicum in vivo and in vitro. Indonesian Journal of Pharmacy, 23(3): 135- 142.

Singh, R.P., Gupta, M. and Agarwal, R. 2008. Silibinun inhibits cororectal cancer growth by inhibiting tumor cell proliferation and angiogenesis. Cancer Research, 68: 2043-2050.

Subramanian, S.S. and Nagarajan, S. 1969: Flavonoids of the seeds of Crotlaria retusa and C. striata. Current Science, 38: $65-68$.

Urquiaga, I. and Leighton, F. 2000. Plant polyphenol, antioxidants and Oxidative stress. Biological Research, 33: 159-165.

Williams, C.A. and Grayer, R.J. 2004. Anthocyanins and other flavonoids. Natural Products Report, 21: 539-573.

Winkel Shirely, B. 2002. Biosynthesis of flavonoids and effects of stress. Current Opinion in Plant Biology, 5: 218-223.

Wong, E. and Francis, C.M. 1968. Flavonoids in genotypes Trifolim subterraneum. I. The normal flavonoids pattern of the geraltton variety. Phytochemistry, 7: 2123-2129.

Watson, A.A., Fleet, G.W.J., Asano, N., Molyneux, R.J. and Nash, R.J. 2001. Polyhydroxylated alkaloids - natural occurrence and therapeutic applications. Phytochemistry, 56: 265-295. 